\title{
Precision medicine for pediatric inflammatory bowel disease: a perspective
}

\section{Yon Ho Choe}

Department of Pediatrics, Samsung Medical Center, Sungkyunkwan University School of Medicine, Seoul, Korea

Received: March 30, 2020

Revised: April 21, 2020

Accepted: April 23, 2020

Corresponding author:

Yon Ho Choe

Department of Pediatrics, Samsung Medical Center, Sungkyunkwan University School of Medicine, 81 Irwonro, Gangnam-gu, Seoul 06351, Korea

Tel: +82-2-3410-3539

E-mail: i101016@skku.edu
This is an Open Access article distributed under the terms of the Creative Commons Attribution Non-Commercial License (https:// creativecommons.org/licenses/ by-nc/4.0/).

\begin{abstract}
There has been a growing need to utilize the concept of "precision medicine" in the treatment of inflammatory bowel disease (IBD). In practice, precision medicine for IBD comprises prediction and personalizing therapies. Prediction is divided into two subcategories: pre-treatment investigation and monitoring response to therapy. Pre-treatment prediction includes clinical outcome assessment, investigation of factors for poor outcome, magnetic resonance enterography, and measurement of TPMT, NUDT15, and other biologic markers. Mucosal healing or transmural healing is considered the "treat-to-target" approach in IBD. To achieve "treat-to-target," the "top-down strategy" is recommended in patients with pediatric Crohn's disease, because the therapeutic window of opportunity may be shorter than that generally believed. We are unable to state that we are truly utilizing biologics in a perfect manner until both "treat-to-target" and "top-down strategy" are supported by "therapeutic drug monitoring (TDM)," a key component of personalizing therapy for IBD. Proactive TDM, or more precisely, model-based proactive dosing with point-of-care assay will soon emerge as the new standard for IBD treatment. It is speculated that "treat-to-target," "top-down strategy," and "TDM" may aid personalized therapy to achieve outstanding improvement in patients with pediatric IBD.
\end{abstract}

Keywords: Biologic products; Drug monitoring; Humans; Inflammatory bowel disease; Precision medicine

\section{INTRODUCTION}

A new era of precision medicine has arrived [1]. Everyone working in the medical field is trying to adopt new initiatives in precision medicine. Precision medicine refers to the "tailoring of medical treatment to the individual characteristics of each patient [2]." Crohn's disease (CD) and ulcerative colitis (UC) have complex pathophysiology including genomes and immune responses, variable clinical manifestations and disease courses, stepwise treatment options, many clinical parameters for monitoring, and unpredictable complications. Therefore, there has been a growing need to utilize the "precision medicine" concept in the treatment of inflammatory bowel disease (IBD).

For optimal "precision medicine" in IBD, precise analysis of the pathophysiology on a case 
by case basis should take precedence. However, the exact pathogenesis of IBD remains unknown. Genetic investigations such as genome-wide association studies reveal only about $25 \%$ of IBD heritability [3]. Even though a recent study on multi-omics of the gut microbiome in IBD provided the most comprehensive descriptions of host and microbial activities in 132 subjects for 1 year, it is still unknown whether dysbiosis is the cause or result of IBD [4]. A study that immunological dysregulation within the gut leading to imbalances in pro- and anti-inflammatory pathways is involved in innate and adaptive immunity showed the possible development and persistence of inflammation in IBD [5]. Approximately $25 \%$ of patients with CD develop symptoms during childhood or adolescence, and pediatric CD is more progressive and extensive than adult-onset CD [6]. Moreover, linear growth impairment is a unique feature of pediatric CD [7]. For the pediatric gastroenterologist, the issue of timing of initiating early biologic treatment is of common interest and one that should be clarified in patients with pediatric IBD [8].

Because of complex disease susceptibility and clinical phenotype, the real-world concept of precision medicine to improve the outcome of patients with pediatric IBD needs to be narrowed down to personalizing therapies by clinicians who attend to these patients. Practical precision medicine in IBD consists of two key areas; one is prediction and the other, personalizing therapies. Prediction is divided into two subcategories: pre-treatment investigation and monitoring response to therapy.

In this article, I focus on the real-world practice of precision medicine in pediatric IBD.

\section{PREDICTION I: PRE-TREATMENT}

\section{Clinical outcome assessment}

In order to select the optimal treatment and predict shortand long-term prognosis, clinicians should be aware of the current status of the patient, based on the objective criteria. Until recently, clinical outcomes have been assessed using the Pediatric Crohn's Disease Activity Index (PCDAI), which is very simple and useful to define severity grading, remission, loss of response, and relapse [9]. It has also been modified as the abbreviated, short, modified, and weighted PCDAl to address various limitations [10]. Because colonoscopic assessment is less acceptable in children, the noninvasive Pediatric Ulcerative Colitis Activity Index (PUCAI) was developed [11]. However, consensus on the definition of successful treatment outcome (clinical response and/or remission) and col- laboration in the development of well-defined and reliable measures of signs and symptoms for use in conjunction with endoscopic parameters of mucosal healing has become necessary to facilitate pediatric IBD drug development [12]. Endoscopy in pediatric IBD provides a more definitive diagnosis and disease extent evaluation, assesses therapeutic efficacy, and leads to a targeted therapy [13]. The reference standard score to evaluate mucosal healing can be measured using the Crohn's Disease Endoscopic Index of Severity (CDEIS), Simple Endoscopic Score for Crohn's Disease (SES-CD), and the Rutgeerts score (for assessing after ileocecal resection) in $\mathrm{CD}$, and the Mayo score, Ulcerative Colitis Endoscopic Index of Severity (UCEIS), and Ulcerative Colitis Colonoscopic Index of Severity (UCCIS) in UC. As a noninvasive surrogate biomarker, fecal calprotectin is available to reveal the current mucosal inflammation and is used in an algorithm for monitoring pediatric UC during the maintenance phase [14].

After clinical outcome assessment, clinicians classify the patients into subgroups. In 2010, pediatric modification and modernization of the Montreal Classification for IBD by the international group of pediatric IBD experts led to the Paris Classification [15]. The Paris Classification includes age at diagnosis, location, behavior, and growth for $\mathrm{CD}$, and extent and severity for UC.

\section{Factors for poor outcome}

According to the consensus guidelines of the European Crohn's and Colitis Organization (ECCO)/European Society of Pediatric Gastroenterology, Hepatology, and Nutrition (ESPGHAN), deep colonic ulceration, persistent severe disease despite adequate induction therapy, extensive (pan-enteric) disease, marked growth retardation, severe osteoporosis, structuring and penetration disease at onset, and severe perianal disease are considered as potentially predictive for poor outcome. These factors may lead to anti-tumor necrosis factor (TNF) therapy as the first-line treatment on an individual basis [16].

\section{TPMT and 6-thioguanine nucleotide}

Thiopurines are recommended as an option for maintenance of steroid-free remission in children at risk for poor disease outcome, and for reducing the formation of antibodies to biologics $[16,17]$. Azathioprine (AZA) is converted to the inactive metabolite 6-methylmercaptopurine by thiopurine S-methyltransferase (TPMT), and patients with inherited TPMT deficiency present with higher 6-thioguanine nucleotide (6-TGN) level and have an increased risk of myelotoxicity [18]. Myelosuppression in patients with IBD treated with thiopurine is re- 
ported more frequently in Korea (31.0\% to $56.4 \%$ ) than in Western countries (2.0\% to $16.7 \%$ ) [18]. According to a Korean report, 137 patients with IBD treated with AZA showed a wild type $\left({ }^{*} 1{ }^{*} 1\right)$ in 130 (94.9\%) patients and variant genotypes in seven patients $\left({ }^{*} 1 /{ }^{*} 3 C\right.$ in four, ${ }^{*} 1 /{ }^{*} 6$ in one, ${ }^{*} 1 /{ }^{*} 16$ in one, and ${ }^{\star} 3 C /{ }^{*} 3 C$ in one) [19]. They also reported that the AZA dosing guidelines for Asian patients with IBD (median AZA dose, 1.01 $\mathrm{mg} / \mathrm{kg} /$ day in this study) may be lower than those of universal guidelines ( 2 to $3 \mathrm{mg} / \mathrm{kg} /$ day in the Clinical Pharmacogenetics Implementation Consortium guidelines [20] and 1.5 to $2.5 \mathrm{mg} / \mathrm{kg} /$ day in the ECCO guidelines [21]).

\section{NUDT15}

In addition to TPMT gene polymorphism, a recent genome-wide association study reported nudix hydrolase 15 (NUDT15) as a novel thiopurine-metabolizing enzyme, a missense variant of which is strongly associated with thiopurine-induced leukopenia, especially in Asians [22]. Previous studies have reported no difference in 6-TGN levels among the NUDT15 genotypes, which implies that NUDT15 variants are independent of 6-TGN levels $[23,24]$. Recently, we conducted a study on NUDT15 polymorphisms in 167 Korean patients who were treated with AZA (unpublished data). Leukopenia was observed in 16\% (19/119), 44\% (20/45), and $100 \%(3 / 3)$ of the NUDT15 normal $\left({ }^{*} 1 /{ }^{*} 1\right)$, intermediate $\left({ }^{\star} 1 /{ }^{\star} 2,{ }^{*} 1 /{ }^{*} 3,{ }^{\star} 1 /{ }^{\star} 5\right.$, and $\left.{ }^{*} 1 /{ }^{*} 6\right)$, and poor $\left({ }^{\star} 3 /{ }^{*} 3\right)$ metabolizer groups, respectively. NUDT15 polymorphism was the only factor associated with time-to-leukopenia (hazard ratio [HR], 4.64; $95 \%$ confidence interval $[\mathrm{Cl}], 2.28$ to $9.45 ; \mathrm{P}<0.001)$. Additional analysis revealed that the 6-TGN levels were significantly higher in patients with leukopenia than those without leukopenia among the 'NUDT15 intermediate+TPMT normal' metabolizer subgroup (median 361.3 vs. $263.8 \mathrm{pmol} / 8 \times 10^{8} \mathrm{RBC}, \mathrm{P}=0.013$ ), indicating that a lower 6-TGN cut-off level is required to avoid leukopenia in this subgroup during AZA treatment. We found that the NUDT15 variant is the most potent factor associated with thiopurine-induced leukopenia and time-to-leukopenia in patients with pediatric IBD during AZA treatment regularly adjusted by thiopurine metabolite monitoring. Therefore, pre-treatment determination of the NUDT15 genotype represents a helpful and effective method for avoiding this life-threatening adverse effect of thiopurines, especially in East Asian pediatric patients.

\section{Anti-Saccharomyces cerevisiae antibodies}

Anti-Saccharomyces cerevisiae antibodies (ASCA) are a useful tool for distinguishing CD from UC [25], and its value as a prognostic marker has been gradually recognized [26]. ASCA is known to be common in younger age groups and those with ileal involvement, fibrostenosis, and more aggressive disease behavior [27]. Several studies have examined whether ASCA titers changed with disease activity, and successful treatment has shown that its titers were stable or decreased $[28,29]$. Recently, our team evaluated the role of ASCA as a predictor of clinical remission and mucosal healing in $61 \mathrm{pa}-$ tients with pediatric IBD (unpublished data). Their ASCA titers maintained similar levels for up to 7 years, and repeated measurements of ASCA after diagnosis are considered unnecessary. In patients who have not achieved mucosal healing, ASCA is closely related with mucosal damage and clinical remission. Therefore, monitoring seems necessary when the titer is initially high. Unlike in Western studies, ASCA immunoglobulin $\mathrm{G}(\mathrm{IgG})$ is more helpful than IgA in predicting prognosis in Korean patients. However, it is speculated that ASCA alone is not enough to be used as an indicator predicting the relapse.

\section{Magnetic resonance enterography}

Recently, magnetic resonance enterography (MRE) and computer tomography enterography have emerged as useful tools for determining small bowel involvement and perianal lesions in $\mathrm{CD}$ [30]. The main advantage of MRE over computer tomography enterography is its lack of ionizing radiation, which is of particular importance when considering small bowel imaging studies in children [31]. The magnetic resonance index of activity (MaRIA) score, a quantitative assessment index based on MRE, was developed and validated for use in the detection of mucosal ulcers and the evaluation of mucosal healing in active $C D$ in adults [32,33]. Our team conducted a study to evaluate 1-year treatment responses to combined immunosuppression using MaRIA with the SES$C D$ as the reference standard in children and adolescents [34]. Therapeutic response assessed by MRE correlated with ileocolonoscopic findings. It was concluded that the baseline wall thickness was lower in mucosa-healed segments 1 year after anti-TNF treatment.

Transmural healing is emerging as a new optimal treatment goal, and we also investigated transmural healing and its relationship with mucosal healing in 72 patients with pediatric $C D$ under maintenance treatment with biologics (unpublished data). Transmural healing was defined as wall thickness $\leq 3 \mathrm{~mm}$ with the absence of ulcers, edema, enhancement, and complications on all ileocolonic segments evaluated by MRE. At 1-year, mucosal healing and transmural healing were achieved in $59.7 \%$ and $22.3 \%$ of the subjects, 
respectively. At 2-year, they were achieved in $66.7 \%$ and $17.2 \%$, respectively. The efforts to achieve transmural healing may alter the natural course of $C D$ in the era of treat-totarget.

\section{REAL WORLD TREATMENT}

\section{Treat-to-target}

The "treat-to-target" approach started from the concept based on the selection of appropriate treatment targets and subsequent adjustment of treatment [35]. In many chronic diseases, reaching an objective target improves long-term outcomes such as low blood pressure in hypertension or low hemoglobin Alc in diabetes. After risk stratification, we specify a target and continue therapy with target surveillance [36]. In IBD, we use this strategy to avoid long-term bowel damage. According to data from large-scale cohort studies and randomized controlled trials, mucosal healing or transmural healing is considered to be the potential treatment target in CD [37-40].

\section{Initiating biologics in pediatric IBD}

The rationale to target early $C D$, especially in children, lies in the fact that they are in the stage of inflammation. They do not yet have fistula, abscess, or stricture. Biologic antibodies to TNF are the strongest anti-inflammatory agents to heal the mucosa. When $C D$ progresses to the fibrostenosing stage, it is more difficult to reverse the bowel damage even with biologics. A recent prospective inception cohort study showed that patients who received early anti-TNF therapy were less likely to have penetrating complications, supporting the usefulness of risk stratification of patients with pediatric $C D$ at diagnosis, and selection of biologics [41].

\section{Preference for the top-down strategy}

There are some reasons why I recommend the top-down strategy in pediatric $C D$. First, it helps better and faster mucosal healing. Kang et al. [42] investigated mucosal healing in patients receiving combo treatment and compared the outcomes between escalation and early treatment. Seventy-eight patients were divided into two groups: the step-up and top-down groups. At 1-year, mucosal healing was better in the top-down group than that in the step-up group. When we investigated the factors associated with mucosal healing at 1 year using multivariate analysis with stepwise selection, the top-down group and mucosal healing at 14 weeks were positively associated with 1-year mucosal healing. Second,

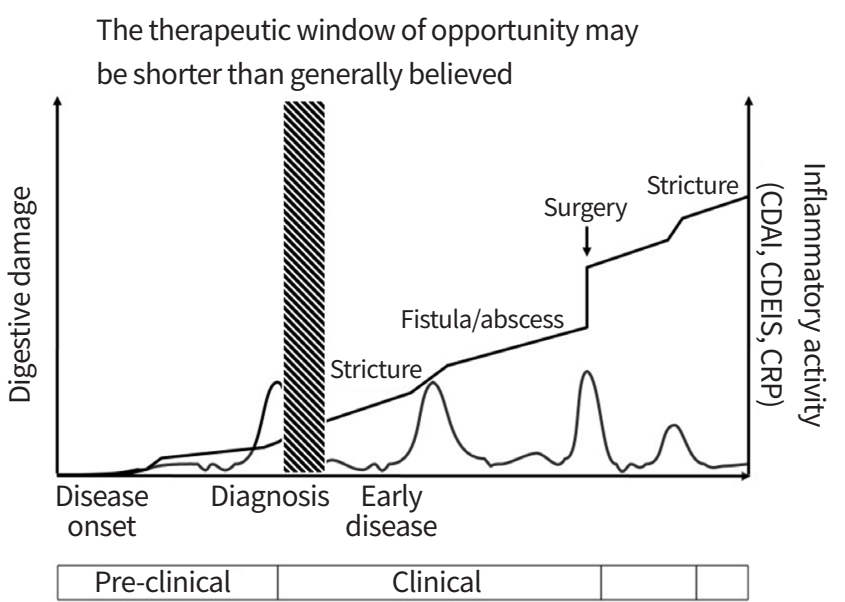

Fig. 1. Progression of digestive damage and inflammatory activity in a theoretical patient with Crohn's disease (CD). The reason "topdown" strategy is recommended in pediatric CD is because the therapeutic window of opportunity may be shorter than generally believed. Adapted from Pariente et al. [45]. CDAl, Crohn's Disease Activity Index; CDEIS, Crohn's Disease Endoscopic Index of Severity; CRP, C-reactive protein.

our team compared long-term remission between the topdown and step-up treatments. The top-down strategy had a longer remission period than that of the step-up strategy in pediatric Crohn disease during the study period of 3 years based on the relapse-free rate and remission period rate [43]. Third, because data comparing the efficacy of the two approaches on growth of children are limited, we investigated the effect of early combined immunosuppression on linear growth in patients with pediatric CD who had sustained remission for 3 years in comparison with those treated by the step-up strategy. The top-down group displayed superior linear growth 3 years after diagnosis $(P=0.026)$. Moreover, a significant difference was also observed in the linear growth of a subgroup at Tanner stages $1-2(P=0.016)$. It was late when the treatment started at Tanner stages 4-5 [44]. Based on the above lines of evidence, I would recommend the top-down strategy, especially in pediatric $C D$, because the therapeutic window of opportunity may be shorter than generally believed (Fig. 1) [45].

\section{PREDICTION II: THERAPEUTIC DRUG MONITORING, A RESPONSE MONITOR- ING DURING MAINTENANCE}

\section{Clinical significance of therapeutic drug monitoring} Assuming that we start treating patients with IBD with biologic antibodies, what would be the issues of most concern? 
The answer must be how to optimize treatment and how to prevent adverse events. For optimal and safe treatment, therapeutic drug monitoring (TDM) is mandatory. TDM provides us not only the important information regarding prediction of patient's response to biologics and/or immunomodulators such as primary non-response, secondary loss of response, remission, relapse, and adverse events during the maintenance of treatment, but also the algorithmic approach in treatment on an individual basis. TDM comprises trough level (TL) and anti-drug antibody measurement. The representative clinical significance of TDM is that high anti-TNF TLs and low anti-drug antibodies are associated with superior clinical and endoscopic outcomes. It is well known that patients who have poor responses and subtherapeutic infliximab (IFX) TLs have an improved response to dose intensification [46]. However, TDM is not yet widely used over the world. CALM study showed that timely escalation with an anti-TNF therapy on the basis of clinical symptoms combined with biomarkers (fecal calprotectin and C-reactive protein) resulted in better clinical and endoscopic outcomes [47].

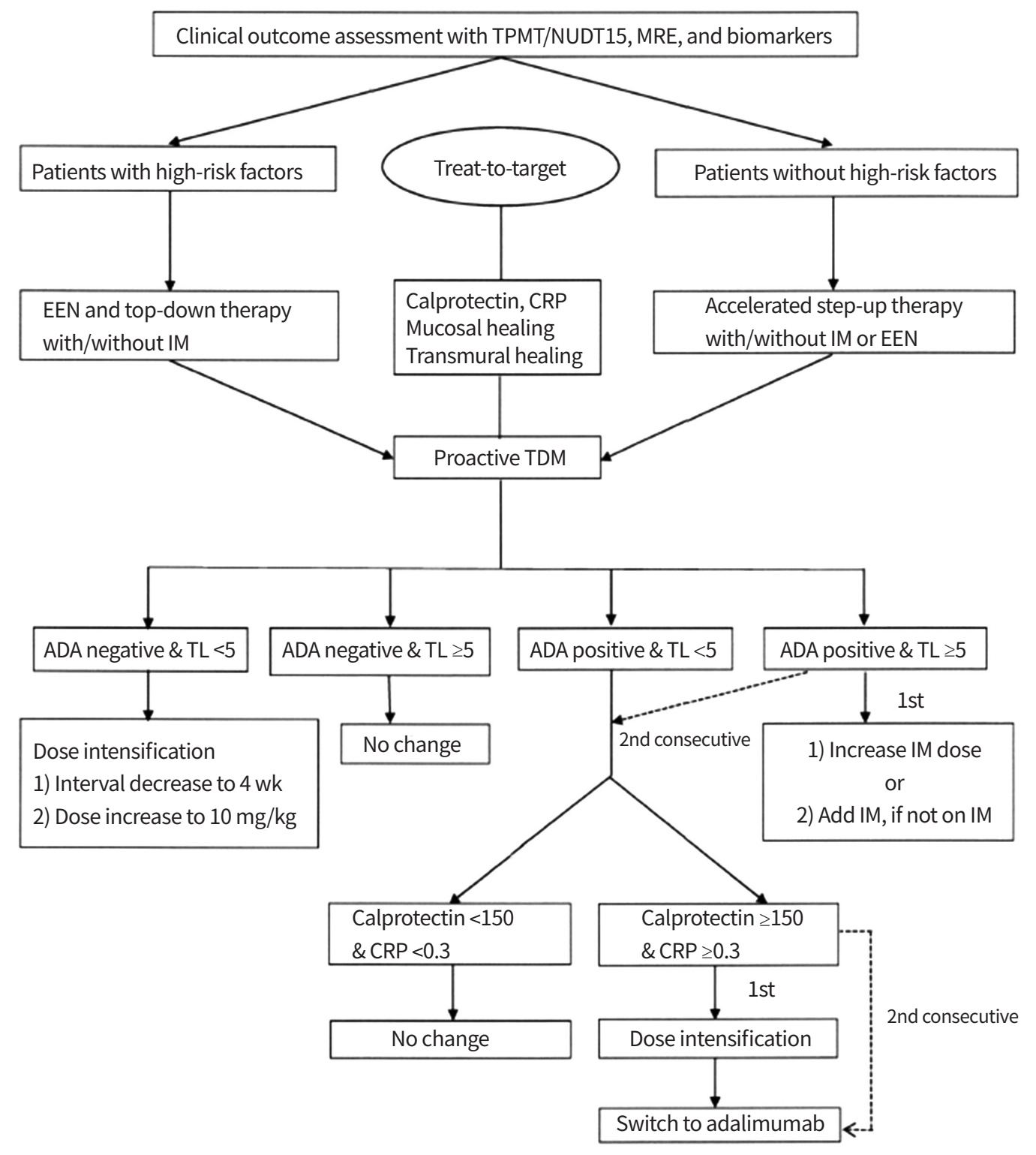

Fig. 2. The Samsung Medical Center protocol of personalizing therapy with infliximab for patients with pediatric Crohn's disease. Measurement units: trough level (TL), $\mu \mathrm{g} / \mathrm{mL}$; anti-drug antibody (ADA), AU/mL; calprotectin, $\mu \mathrm{g} / \mathrm{g}$; C-reactive protein (CRP), mg/dL. Highrisk factors: deep colonic ulcerations, extensive disease, marked growth retardation, severe osteoporosis, B2 and/or B3 behavior, severe perianal disease. Adapted from Ruemmele et al. [16]. TPMT, thiopurine S-methyltransferase; NUDT15, nudix hydrolase 15; MRE, magnetic resonance enterography; EEN, exclusive enteral nutrition; IM, immunomodulator; TDM, therapeutic drug monitoring. 


\section{Reactive vs. proactive TDM}

Reactive TDM is widely accepted in practice at the time of loss of response, especially for anti-TNF antibodies. Besides reactive testing, there is a movement toward proactively adjusting biologic dosing to prevent loss of response, in keeping with the tight control philosophy of IBD care [48]. Although the American Gastroenterological Association advocates reactive TDM in adults with active IBD failing response to anti-TNF therapy in a recent guideline regarding TDM, Papamichael et al. [49] contradict this statement. They mention that it is more rational to proactively optimize anti-TNF therapy before immunogenicity and/or loss of response, because proactive TDM is a standard of care for many medical therapies such as anticoagulants, antibiotics, and transplantation [50]. Our team at the Samsung Medical Center is also using the proactive TDM protocol in pediatric patients with moderate-to severe CD (Fig. 2). As worldwide data accumulate, it is expected that proactive TDM will emerge as the standard IBD treatment.

\section{De-escalation}

There are currently insufficient data to recommend when and in whom discontinuing anti-TNF treatment is suitable among patients with $\mathrm{CD}$, and in clinical practice, the decision of whether to continue biologics is typically based on weighing the risks and benefits for each patient on an individual basis [51]. According to clinicians who treat patients with $\mathrm{IBD}$, we are concerned regarding loss of response or relapse during maintenance; therefore, doctors prefer the long-term use of current biologics. However, if I were a patient or a parent of the patient who is in the quiescent phase, I would consider cessation of the biologic antibodies and/or immunomodulators because of the possible adverse events after long-term use. According to a STORI trial, an IFX TL of $\geq 2 \mu \mathrm{g} /$ $\mathrm{mL}$ was associated with relapse after IFX cessation (HR, 2.5; $95 \% \mathrm{Cl}, 1.1$ to 5.4; $\mathrm{P}=0.02$ ) [52]. Meanwhile, Papamichael et al. [53] reported that an IFX TL of $<6 \mu \mathrm{g} / \mathrm{mL}$ was associated with sustained clinical remission after IFX cessation. We also conducted a study on subtherapeutic IFX TLs and complete mucosal healing associated with sustained clinical remission after IFX cessation in patients with pediatric CD. Patients were followed for up to 7 years after IFX cessation and the sustained clinical remission rate was high when IFX was stopped at TL under $2.5 \mu \mathrm{g} / \mathrm{mL}$ and complete mucosal healing was achieved [51]. It is speculated that there may be a subgroup of patients who are good candidates for IFX withdrawal, even though IFX cessation in patients with pediatric $C D$ remains inadvisable at present.

\section{Point-of-care assay}

Point-of-care (POC) assays are analytical devices that can be performed on-site for TDM by clinical staff without laboratory training and ideally without the need for sample transportation and preparation, thereby decreasing the turnaround time. POC assay is quite promising in optimizing the drug dosage based on real-time pharmacokinetic information without a delay in information by weeks to months as previously [48]. If the whole blood can be applied technically for assay instead of the serum, on-site use of POC TDM will become widespread. While not currently available for all biologics, POC TDM is warranted in certain circumstances such as acute severe colitis and enables personalized, precision dosing [54].

\section{NEW CONCEPT OF IBD TREATMENT}

\section{Optimized monotherapy}

Although current data have suggested that combination therapy is superior to IFX monotherapy, a retrospective study showed that optimizing IFX to a TL of $\geq 5 \mu \mathrm{g} / \mathrm{mL}$ may provide an alternative treatment strategy to combination therapy, suggesting proactive TDM can potentially guide immunomodulatory withdrawal in patients on combination therapy [55]. A recent study further supported the concept of "optimized monotherapy" by showing that IFX durability did not differ between patients on IFX monotherapy doses based on proactive TDM and patients receiving combination therapy, if the first maintenance infusion is dosed based on week 10 proactive TDM [56].

\section{Dual biologic treatment}

Anti-TNF antibodies are widely used biologic agents for IBD treatment, and vedolizumab is a monoclonal antibody against $\mathrm{a} 4 \mathrm{~b} 7$, an integrin facilitating leucocyte migration to the gut via binding of MADCAM1 on the intestinal endothelium. A gut-specific anti-integrin therapy like vedolizumab has the benefit of being potentially safer than systemic therapies and could be the ideal drug for concomitant use with other target therapies [57]. Given its mechanism of action, it seems reasonable to postulate that vedolizumab could be safely combined with other biologics, without increasing the risk of serious infections [57]. Through a systematic review including seven studies with 18 patients who received dual biologics, Ribaldone et al. [57] reported that a clinical improvement was obtained in $100 \%$ of patients and an endoscopic improvement in $93 \%$ of patients. No serious adverse events 
were reported. They concluded that the use of dual biological therapy is an attractive therapeutic option and may be an opportunity to better tailor and personalize the therapies for patients [57].

\section{Model-informed precision dosing}

Even though reactive or proactive TDM algorithms have proposed optimal dosing strategies to guide rational decision making, there are still numerous cases with unpredictable pharmacokinetic variability, which confuses clinical practitioners, thereby undermining the benefits of TDM. Model-informed precision dosing (MIPD) has emerged as a strategy to overcome this problem. A population pharmacokinetic model is the basis of MIPD and serves as a Bayesian prior [48]. Based on patient characteristics and drug concentration measurements, the individual pharmacokinetic parameters can be calculated (empiric Bayes estimates) and subsequently be used to predict the next dose that is required for that patient to achieve a predefined exposure target [48]. Strik et al. [58] conducted a study to compare the efficacy of model-based dose optimization of IFX TL $3.0 \mu \mathrm{g} / \mathrm{mL}$ with that of a control group that continued IFX dosing without dose adaptation in the PRECISION trial. They revealed that a higher proportion of patients significantly maintained clinical remission when on model-based TDM.

\section{CONCLUSION}

This review discusses the real-world practice of patients with pediatric IBD from pre-treatment prediction to maintenance therapy with TDM, and suggests future treatment strategy based on the current possible approach of precision medicine. The "treat-to-target" concept gave birth to the "topdown strategy," especially in pediatric IBD, even though each of these started from different branches of medicine. There has been a trend that both have converged during the past decade. We are unable to state that we are truly utilizing biologics in a perfect manner until both the "treat-to-target" and "top-down strategy" are supported by "TDM," a key component of personalizing therapy for IBD. Proactive TDM, more precisely, model-based proactive dosing with $\mathrm{POC}$ assay will soon emerge as the new standard for IBD treatment. In conclusion, it is speculated that "treat-to-target," "top-down strategy," and "TDM" are in the same context of personalizing therapy to achieve outstanding improvement in patients with IBD.

\section{CONFLICTS OF INTEREST}

No potential conflict of interest relevant to this article was reported.

\section{ORCID}

Yon Ho Choe https://orcid.org/0000-0003-1525-7688

\section{AUTHOR CONTRIBUTIONS}

Conception or design: YHC.

Acquisition, analysis, or interpretation of data: YHC.

Drafting the work or revising: YHC.

Final approval of the manuscript: YHC.

\section{REFERENCES}

1. Collins FS, Varmus H. A new initiative on precision medicine. N Engl J Med 2015;372:793-5.

2. National Research Council (US) Committee on A Framework for Developing a New Taxonomy of Disease. Toward precision medicine: building a knowledge network for biomedical research and a new taxonomy of disease. Washington (DC): National Academies Press; 2011.

3. McGovern DP, Kugathasan S, Cho JH. Genetics of inflammatory bowel diseases. Gastroenterology 2015;149:116376.

4. Lloyd-Price J, Arze C, Ananthakrishnan AN, Schirmer M, Avila-Pacheco J, Poon TW, et al. Multi-omics of the gut microbial ecosystem in inflammatory bowel diseases. Version 2. Nature 2019;569:655-62.

5. Park JH, Peyrin-Biroulet L, Eisenhut M, Shin JI. IBD immunopathogenesis: a comprehensive review of inflammatory molecules. Autoimmun Rev 2017;16:416-26.

6. Rosen MJ, Dhawan A, Saeed SA. Inflammatory bowel disease in children and adolescents. JAMA Pediatr 2015;169: 1053-60.

7. Walters TD, Griffiths AM. Mechanisms of growth impairment in pediatric Crohn's disease. Nat Rev Gastroenterol Hepatol 2009;6:513-23.

8. Kang B, Choe YH. Early biologic treatment in pediatric Crohn's disease: catching the therapeutic window of opportunity in early disease by treat-to-target. Pediatr Gastroenterol Hepatol Nutr 2018;21:1-11.

9. Hyams JS, Ferry GD, Mandel FS, Gryboski JD, Kibort PM, Kirschner BS, et al. Development and validation of a pe- 
diatric Crohn's disease activity index. J Pediatr Gastroenterol Nutr 1991;12:439-47.

10. Sun H, Vesely R, Lee KJ, Klein A, Ikima M, Mulberg AE, et al. Pediatric Crohn disease clinical outcome assessments and biomarkers: current state and path forward for global collaboration. J Pediatr Gastroenterol Nutr 2017;64:36872 .

11. Turner D, Otley AR, Mack D, Hyams J, de Bruijne J, Uusoue $\mathrm{K}$, et al. Development, validation, and evaluation of a pediatric ulcerative colitis activity index: a prospective multicenter study. Gastroenterology 2007;133:423-32.

12. Sun H, Lee JJ, Papadopoulos EJ, Lee CS, Nelson RM, Sachs HC, et al. Alternate endpoints and clinical outcome assessments in pediatric ulcerative colitis registration trials. J Pediatr Gastroenterol Nutr 2014;58:12-7.

13. Oliva S, Thomson M, de Ridder L, Martin-de-Carpi J, Van Biervliet S, Braegger C, et al. Endoscopy in pediatric inflammatory bowel disease: a position paper on behalf of the Porto IBD group of the ESPGHAN. J Pediatr Gastroenterol Nutr 2018;67:414-30.

14. Turner D, Ruemmele FM, Orlanski-Meyer E, Griffiths AM, de Carpi JM, Bronsky J, et al. Management of paediatric ulcerative colitis, part 1: ambulatory care-an evidence-based guideline from European Crohn's and Colitis Organization and European Society of Paediatric Gastroenterology, Hepatology and Nutrition. J Pediatr Gastroenterol Nutr 2018;67:257-91.

15. Levine A, Griffiths A, Markowitz J, Wilson DC, Turner D, Russell RK, et al. Pediatric modification of the Montreal classification for inflammatory bowel disease: the Paris classification. Inflamm Bowel Dis 2011;17:1314-21.

16. Ruemmele FM, Veres G, Kolho KL, Griffiths A, Levine A, Escher JC, et al. Consensus guidelines of ECCO/ESPGHAN on the medical management of pediatric Crohn's disease. J Crohns Colitis 2014;8:1179-207.

17. Bar-Yoseph H, Waterman M, Almog R, Billiet T, Vermeire S, Ungar B, et al. Prevention of antidrug antibody formation to infliximab in Crohn's patients with prior failure of thiopurines. Clin Gastroenterol Hepatol 2017;15:69-75.

18. Lee MN, Kang B, Choi SY, Kim MJ, Woo SY, Kim JW, et al. Impact of genetic polymorphisms on 6-thioguanine nucleotide levels and toxicity in pediatric patients with IBD treated with azathioprine. Inflamm Bowel Dis 2015;21:2897-908.

19. Lee MN, Kang B, Choi SY, Kim MJ, Woo SY, Kim JW, et al. Relationship between azathioprine dosage, 6-thioguanine nucleotide levels, and therapeutic response in pediatric patients with IBD treated with azathioprine. In- flamm Bowel Dis 2015;21:1054-62.

20. Relling MV, Gardner EE, Sandborn WJ, Schmiegelow K, Pui CH, Yee SW, et al. Clinical Pharmacogenetics Implementation Consortium guidelines for thiopurine methyltransferase genotype and thiopurine dosing. Version 2. Clin Pharmacol Ther 2011;89:387-91.

21. Dignass A, Van Assche G, Lindsay JO, Lemann M, Soderholm J, Colombel JF, et al. The second European evidence-based consensus on the diagnosis and management of Crohn's disease: current management. J Crohns Colitis 2010;4:28-62.

22. Yang SK, Hong M, Baek J, Choi H, Zhao W, Jung Y, et al. A common missense variant in NUDT15 confers susceptibility to thiopurine-induced leukopenia. Nat Genet 2014; 46:1017-20.

23. Zhu X, Wang XD, Chao K, Zhi M, Zheng H, Ruan HL, et al. NUDT15 polymorphisms are better than thiopurine S-methyltransferase as predictor of risk for thiopurine-induced leukopenia in Chinese patients with Crohn's disease. Aliment Pharmacol Ther 2016;44:967-75.

24. Asada A, Nishida A, Shioya M, Imaeda H, Inatomi O, Bamba S, et al. NUDT15 R139C-related thiopurine leukocytopenia is mediated by 6 -thioguanine nucleotide-independent mechanism in Japanese patients with inflammatory bowel disease. J Gastroenterol 2016;51:22-9.

25. Main J, McKenzie H, Yeaman GR, Kerr MA, Robson D, Pennington CR, et al. Antibody to Saccharomyces cerevisiae (bakers' yeast) in Crohn's disease. BMJ 1988;297:1105-6.

26. Lewis JD. The utility of biomarkers in the diagnosis and therapy of inflammatory bowel disease. Gastroenterology 2011;140:1817-26.

27. Zhang Z, Li C, Zhao X, Lv C, He Q, Lei S, et al. Anti-Saccharomyces cerevisiae antibodies associate with phenotypes and higher risk for surgery in Crohn's disease: a meta-analysis. Dig Dis Sci 2012;57:2944-54.

28. Teml A, Kratzer V, Schneider B, Lochs H, Norman GL, Gangl A, et al. Anti-Saccharomyces cerevisiae antibodies: a stable marker for Crohn's disease during steroid and 5-aminosalicylic acid treatment. Am J Gastroenterol 2003;98:2226-31.

29. Oshitani N, Hato F, Matsumoto T, Jinno Y, Sawa Y, Hara J, et al. Decreased anti-Saccharomyces cerevisiae antibody titer by mesalazine in patients with Crohn's disease. J Gastroenterol Hepatol 2000;15:1400-3.

30. Amitai MM, Ben-Horin S, Eliakim R, Kopylov U. Magnetic resonance enterography in Crohn's disease: a guide to common imaging manifestations for the IBD physician. J Crohns Colitis 2013;7:603-15. 
31. Gaca AM, Jaffe TA, Delaney S, Yoshizumi T, Toncheva G, Nguyen G, et al. Radiation doses from small-bowel follow-through and abdomen/pelvis MDCT in pediatric Crohn disease. Pediatr Radiol 2008;38:285-91.

32. Rimola J, Rodriguez S, Garcia-Bosch O, Ordas I, Ayala E, Aceituno $\mathrm{M}$, et al. Magnetic resonance for assessment of disease activity and severity in ileocolonic Crohn's disease. Gut 2009;58:1113-20.

33. Ordas I, Rimola J, Rodriguez S, Paredes JM, Martinez-Perez MJ, Blanc E, et al. Accuracy of magnetic resonance enterography in assessing response to therapy and mucosal healing in patients with Crohn's disease. Gastroenterology 2014;146:374-82.

34. Kang B, Choi SY, Chi S, Lim Y, Jeon TY, Choe YH. Baseline wall thickness is lower in mucosa-healed segments 1 year after infliximab in pediatric Crohn disease patients. J Pediatr Gastroenterol Nutr 2017;64:279-85.

35. van Vollenhoven R. Treat-to-target in rheumatoid arthritis: are we there yet? Nat Rev Rheumatol 2019;15:180-6.

36. Bouguen G, Levesque BG, Feagan BG, Kavanaugh A, Peyrin-Biroulet L, Colombel JF, et al. Treat to target: a proposed new paradigm for the management of Crohn's disease. Clin Gastroenterol Hepatol 2015;13:1042-50.

37. Rutgeerts P, Feagan BG, Lichtenstein GR, Mayer LF, Schreiber S, Colombel JF, et al. Comparison of scheduled and episodic treatment strategies of infliximab in Crohn's disease. Gastroenterology 2004;126:402-13.

38. Baert F, Moortgat L, Van Assche G, Caenepeel P, Vergauwe $P$, De Vos M, et al. Mucosal healing predicts sustained clinical remission in patients with early-stage Crohn's disease. Gastroenterology 2010;138:463-8.

39. Froslie KF, Jahnsen J, Moum BA, Vatn MH; IBSEN Group. Mucosal healing in inflammatory bowel disease: results from a Norwegian population-based cohort. Gastroenterology 2007;133:412-22.

40. Serban ED. Treat-to-target in Crohn's disease: will transmural healing become a therapeutic endpoint? World J Clin Cases 2018;6:501-13.

41. Kugathasan S, Denson LA, Walters TD, Kim MO, Marigorta UM, Schirmer M, et al. Prediction of complicated disease course for children newly diagnosed with Crohn's disease: a multicentre inception cohort study. Lancet 2017; 389:1710-8.

42. Kang B, Choi SY, Kim HS, Kim K, Lee YM, Choe YH. Mucosal healing in paediatric patients with moderate-to-severe luminal Crohn's disease under combined immunosuppression: escalation versus early treatment. J Crohns
Colitis 2016;10:1279-86.

43. Lee YM, Kang B, Lee Y, Kim MJ, Choe YH. Infliximab "topdown" strategy is superior to "step-up" in maintaining long-term remission in the treatment of pediatric Crohn disease. J Pediatr Gastroenterol Nutr 2015;60:737-43.

44. Choi J, Kang B, Kim MJ, Sohn I, Lee HJ, Choe YH. Early infliximab yields superior long-term effects on linear growth in pediatric Crohn's disease patients. Gut Liver 2018;12:255-62.

45. Pariente B, Cosnes J, Danese S, Sandborn WJ, Lewin M, Fletcher JG, et al. Development of the Crohn's disease digestive damage score, the Lemann score. Inflamm Bowel Dis 2011;17:1415-22.

46. Choi SY, Kang B, Lee JH, Choe YH. Clinical use of measuring trough levels and antibodies against infliximab in patients with pediatric inflammatory bowel disease. Gut Liver 2017;11:55-61.

47. Colombel JF, Panaccione R, Bossuyt P, Lukas M, Baert F, Vanasek $T$, et al. Effect of tight control management on Crohn's disease (CALM): a multicentre, randomised, controlled phase 3 trial. Lancet 2018;390:2779-89.

48. Vermeire S, Dreesen E, Papamichael K, Dubinsky MC. How, when, and for whom should we perform therapeutic drug monitoring? Clin Gastroenterol Hepatol 2020;18:1291-9.

49. Feuerstein JD, Nguyen GC, Kupfer SS, Falck-Ytter Y, Singh S; American Gastroenterological Association Institute Clinical Guidelines Committee. American Gastroenterological Association Institute guideline on therapeutic drug monitoring in inflammatory bowel disease. Gastroenterology 2017;153:827-34.

50. Papamichael K, Osterman MT, Siegel CA, Melmed GY, Dubinsky MC, Colombel JF, et al. Using proactive therapeutic drug monitoring of anti-tumor necrosis factor therapy in inflammatory bowel disease: from an old concept to a future standard of care? Gastroenterology 2018;154:12012.

51. Kang B, Choi SY, Choi YO, Kim MJ, Kim K, Lee JH, et al. Subtherapeutic infliximab trough levels and complete mucosal healing are associated with sustained clinical remission after infliximab cessation in paediatric-onset Crohn's disease patients treated with combined immunosuppressive therapy. J Crohns Colitis 2018;12:644-52.

52. Louis E, Mary JY, Vernier-Massouille G, Grimaud JC, Bouhnik Y, Laharie D, et al. Maintenance of remission among patients with Crohn's disease on antimetabolite therapy after infliximab therapy is stopped. Gastroenterology 2012;142:63-70. 
53. Papamichael K, Vande Casteele N, Gils A, Tops S, Hauenstein S, Singh S, et al. Long-term outcome of patients with Crohn's disease who discontinued infliximab therapy upon clinical remission. Clin Gastroenterol Hepatol 2015;13:1103-10.

54. Strik AS, Wang YC, Ruff LE, Yashar W, Messmer BT, Mould DR. Individualized dosing of therapeutic monoclonal antibodies: a changing treatment paradigm? AAPS J 2018; 20:99.

55. Vaughn BP, Martinez-Vazquez M, Patwardhan VR, Moss AC, Sandborn WJ, Cheifetz AS. Proactive therapeutic concentration monitoring of infliximab may improve outcomes for patients with inflammatory bowel disease: results from a pilot observational study. Inflamm Bowel Dis 2014;20:19962003.
56. Lega S, Phan BL, Rosenthal CJ, Gordon J, Haddad N, Pittman N, et al. Proactively optimized infliximab monotherapy is as effective as combination therapy in IBD. Inflamm Bowel Dis 2019;25:134-41.

57. Ribaldone DG, Pellicano R, Vernero M, Caviglia GP, Saracco GM, Morino M, et al. Dual biological therapy with anti-TNF, vedolizumab or ustekinumab in inflammatory bowel disease: a systematic review with pool analysis. Scand J Gastroenterol 2019;54:407-13.

58. Strik A, Berends S, Mould D, Mathot R, Ponsioen C, van den Brande J, et al. DOP56 Dashboard driven vs. conventional dosing of infliximab in inflammatory bowel disease patients: the PRECISION trial. J Crohns Colitis 2019;13(Suppl 1):S063. 